\title{
Dielectric Dispersion of Hinged-PBLG (Head to Head)
}

\author{
Hiroshi Kinara, ${ }^{*}$ Kayoko TANno, and Akiyoshi WADA \\ Department of Physics, Faculty of Science, The \\ University of Tokyo, Tokyo 113, Japan.
}

(Received November 24, 1972)

\author{
KEY WORDS Poly( $\gamma$-benzyl glutamate) / Dielectric Dispersion / \\ Hinged-Polymer / Helix-Coil Transition /
}

The Dielectric dispersion of hinged polypeptides in solution has been studied in order to make clear the interaction of the helical parts in an interrupted helix. Since such molecules are composed of two $\alpha$-helices jointed by a coiling part of the bifunctional initiator used in the polymerization, they can be considered as models for the broken helices which appear in the intermediate states of the helix-coil transition and in the folding of $\alpha$-helices in protein molecules.

Hinged poly( $\gamma$-benzyl-L-glutamate) (hinged PBLG) samples were synthesized in dioxane by polymerizing an $\mathrm{N}$-carboxyanhydride of $\gamma$-benzylL-glutamate, with trimethylenediamine (TMDA $)^{1,2}$ and hexamethylenediamine (HMDA) as initiators. The HMDA initiation was found to be successful only when carried out in a nitrogen atmosphere. Sample 7010 was fractionated into four parts (coded 7010 A, B, C and D) in a dichloromethane (DCM)-methanol system. The molecular weights of the samples thus obtained were determined from their intrinsic viscosities in dichloroacetic acid (DCA) solution. ${ }^{3}$ The relevant data on the samples used in the present experiments are listed in Table I.

Dielectric measurements were performed on both usual and hinged PBLG dissolved in $m$ cresol. Use was made of a transformer bridge TR-10C (Ando Denki Co., Tokyo) ${ }^{4}$ with a cylindrical cell of air capacitance $2.92_{5} \mathrm{pF}$. $m$ Cresol was fractionally distilled under reduced pressure, after dehydration with phosphorous pentoxide. The temperature of each solution was adjusted within $\pm 0.01^{\circ} \mathrm{C}$ of the desired value.

Figure 1 shows the concentration dependence of the specific polarization reduced to $25^{\circ} \mathrm{C}$ for sample $7010 \mathrm{~B}$ in $\mathrm{m}$-cresol, which indicates that no marked aggregations existed. From the figure, a value of $232 \pm 12(\mathrm{~m} l / \mathrm{g})$ is obtaind for the specific polarization of $7010 \mathrm{~B}$ reduced to the standard state of $25^{\circ} \mathrm{C}$ at infinite dilution.

Figure 2 illustrates how the specific polarization in $m$-cresol varies with molecular weight for the two types of PBLG. If the sample is a single perfect $\alpha$-helix, it should have a value of 3.4D for the dipole moment per monomeric re-

Table I. Relevant data for hinged-PBLG samples

\begin{tabular}{cccclcc}
\hline Sample code, & \multicolumn{2}{c}{$A / I$ Conc. of NCA } & Solvent Ext. of react. & {$[\eta]_{\mathrm{DCA}}$} & $M_{w}$ & Initiator \\
\hline $7010 \mathrm{~B}$ & 135 & $2 \%(\mathrm{G} / \mathrm{ML})$ & Dioxane $54.5 \%$ & $0.69(\mathrm{DL} / \mathrm{G})$ & $11.2 \times 10^{4}$ & TMDA \\
$7010 \mathrm{C}$ & 135 & 2 & Dioxane 54.5 & 0.36 & 5.3 & TMDA \\
71091 & $202 \sim 2$ & Dioxane & 0.34 & 5.0 & TMDA \\
$7003^{\mathrm{a}}$ & 13 & 6 & Dioxane 96 & 0.48 & 7.41 & $\mathrm{NaOMe}$ \\
\hline
\end{tabular}

a Sample of the single $\alpha$-helix used for reference.

\footnotetext{
* Present address: Department of Physics, Jichi Medical School, Yakushiji, Kawachi-gun, Tochigipref. 329-04 Japan.
} 


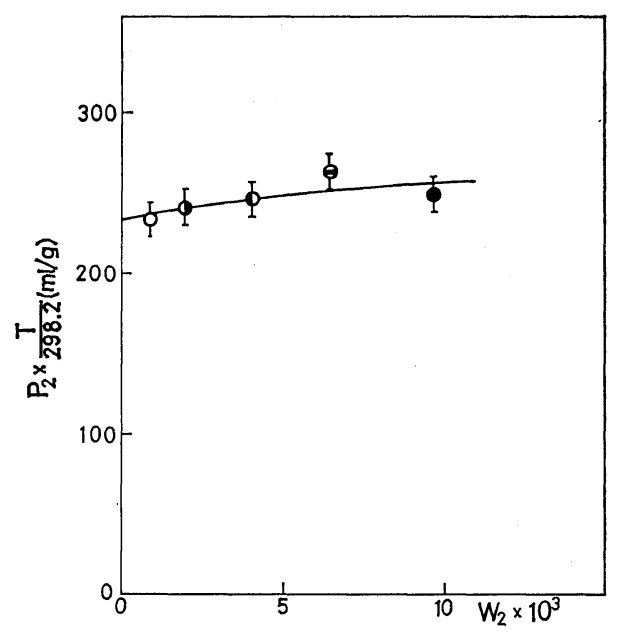

Figure 1. The concentration dependence of the specific polarization. The sample is 7010B: (O), $T=84.5^{\circ} \mathrm{C} ;(\bigcirc), T=25^{\circ} \mathrm{C} ;(\bigcirc), T=71^{\circ} \mathrm{C} ;(\ominus), 68^{\circ} \mathrm{C}$; (O), $T=69^{\circ} \mathrm{C} ;\left(T\right.$. Temp, $P_{2}$, specific polarization of the solute, $W_{2}$; weight fraction of the solute).

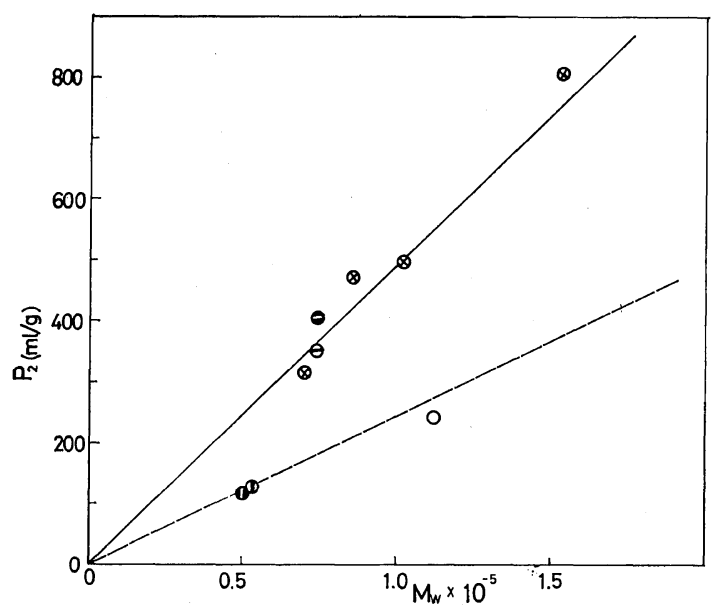

Figure 2. The molecular weight dependence of specific polarization. The real line represents the single perfect $\alpha$-helix $\left(\mu_{0}=3.4 \mathrm{D} /\right.$ residue $)$. The broken line represents the case of no interactions between helices $\left(\theta=90^{\circ}\right)$. (O), 7010B; (D), 7010C; (D), 71091; $(\ominus), 7003$ in $m$-cresol. (Ө), 7003 in EDC-DMF solution. (®), The data measured by $\mathrm{Wada}^{5}$ in EDC solution, presented for comparison.

sidue. $^{5, *}$ The solid line in the figure represents

* T. Matsumoto, et al., reported a value slightly higher than $3.4 \mathrm{D}$. this relation, and the corresponding data points are seen to follow it nicely. On the other hand, if the samples have a highly flexible hinge and no interactions between the two helices separated by it exist, the data should be on the broken line of $\theta=90^{\circ}$, where $\theta$ is the average angle between two helices. The specific polarizations of the hinged polymers were found to be a little below this line. The deviation seems to be reasonable when the electrostatic interaction (repulsive in this case) between helices is taken into account.

We now, discuss the flexibility of the coiling part, noting several points. First, a slight positive increase of the specific polarization was found in the temperature range between $25^{\circ} \mathrm{C}$ and $75^{\circ} \mathrm{C}$ (Figure 3). This behavior agrees with the temperature dependence calculated by taking the electrostostatic interaction between helices into account. Second, since the polymers from TMDA-initiation and HMDA-initiation, which have different lengths for the flexible parts, give similar results, both polymers may have hinges of similar flexibilities or they may show a different dependence upon the molecular weight. Third, the energy map of the coiling part as a function of $\theta$ was calculated from the data of $n$-butane by Flory ${ }^{5}$ and those of the butyl residue in dipeptide unit from the data by Ponnuswamy, et $a l .{ }^{6}$ The map shows that even in the TMDA

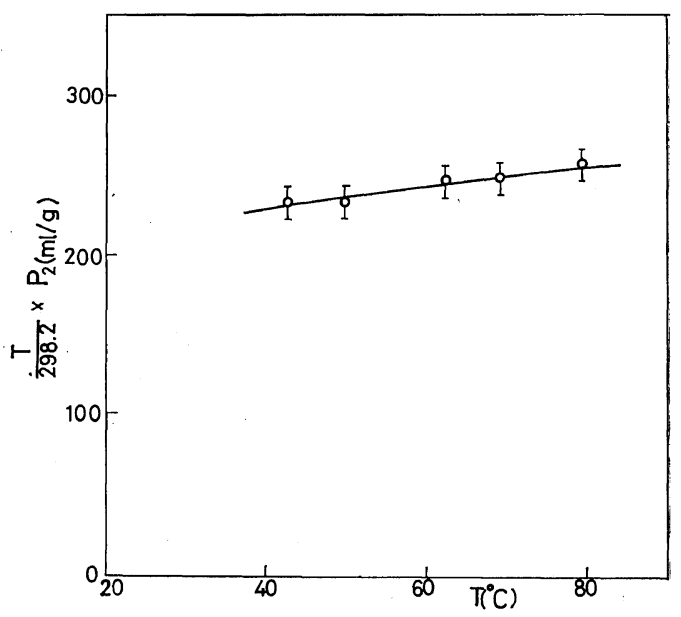

Figure 3. Temperature dependence of the specific polarizations. The sample is $7010 \mathrm{~B} . W_{2}$ is $9.66 \times$ $10^{-3}$. 
hinge a chain segment $-\mathrm{NH}-\mathrm{CH}-\mathrm{CO}-\mathrm{NH}-$

$\mathrm{CH}_{2} \mathrm{C}_{6} \mathrm{H}_{5}$ ) is enough to join two helices, with a potential barrier of less than one kcal. Furthermore, no serious steric hinderance of long-range was encountered when the molecular model of the hinged part was constructed and rotated in all directions.

The arguments presented above lead us to conclude that the coiling part is perfectly or nearly perfectly flexible at room temperature, and that the electrostatic repulsive force between $\alpha$-helices jointed in the head-to-head manner has only a small effect on the joint angle.

Acknowledgement. The authors wish to thank Professor A. Teramoto for useful suggestions and discussions.

\section{REFERENCES}

1. A. Teramoto, T. Yamashita, and H. Fujita, $J$. Chem. Phys., 46, 1919 (1967).

2. K. Nakagawa, N. Nishioka, A. Teramoto, and H. Fujita, Polymer J., 4, 332 (1973).

3. R. D. Lundberg and P. Doty, J. Amer. Chem. Soc., 78, 941 (1956).

4. A. Wada, Poly- $\alpha$-Amino Acids, Chap. 9, G. Fasman ed., Marcel Dekker, New York, N. Y., 1968.

5. P. J. Flory, Statistical Mechanics of Chain Molecules, Interscience Publisher, Inc., New York, N. Y., 1969.

6. P. K. Ponnuswamy and V. Sasisekharan, Biopolymers, 10, 565 (1971).

7. T. Matsumoto, A. Teramoto, and H. Fujita, report read at the SPSJ 21th Symposium on Macromolecules Osaka, Japan, 1972. 